J Ästhet Chir 2021 · 14:108-112

https://doi.org/10.1007/s12631-021-00266-w

Angenommen: 8. April 2021

Online publiziert: 11 . Mai 2021

(c) Springer Medizin Verlag GmbH, ein Teil von Springer Nature 2021

\section{Redaktion}

F.G. Neidel, Düsseldorf

\title{
Annette Hortling
}

SIEGAESTHETIK, Praxis für Ästhetische und Plastische Chirurgie, Haarchirurgie, Siegburg, Deutschland

\section{Gutachten nach Haartransplantation}

\section{Auf was sollte man in der präoperativen Aufklärung achten?}

Trotz des deutlichen Benefits der BeLaut des internationalen Haarverbandes ISHRS (International Society of Hair Restoration Surgery) wurden 2017 weltweit 636.000 Haartransplantationen durchgeführt [1]. Dabei ist bei zunehmender Nachfrage und Akzeptanz für diese korrektiven Maßnahmen ein deutlich ansteigender Trend zu beobachten. Hierzu haben sicherlich auch die veröffentlichten Fälle prominenter Persönlichkeiten mit sehr guten Ergebnissen beigetragen.

Bei einer Haartransplantation werden die zu transplantierenden Haarwurzeln meist aus dem Hinterkopfbereich entnommen und an die gewünschte Stelle im Oberkopf-, Augenbrauen- oder Bartbereich wieder implantiert.

Insbesondere die Eigenhaartransplantation (HTX) in „Follicular-unitexcision“(FUE)-Technik scheint sich als wenig invasive Methode im Vergleich zur „Follicular-unit-transplantation" (FUT) mit Entnahme eines Hautstreifens vom Hinterkopf zahlenmäßig durchzusetzen. Die Operationsergebnisse und damit die Patientenzufriedenheit sind dabei unter Beachtung der bestehenden Operationsstandards als sehr zufriedenstellend zu bezeichnen. Das Risiko für Komplikationen und dauerhafte Schäden am Patienten ist entsprechend einem Eingriff an der Körperoberfläche gering.

Dabei stellt eine Haartransplantation sehr hohe Ansprüche an die Detailarbeit des Haarchirurgen und dessen Assistenzpersonal. Eine erfolgreiche HTX mit natürlichem postoperativem Haarbild und Haardichte erfordert ein hohes Maß an Perfektion und Ausdauervermögen der Agierenden. handlung kommt es hin und wieder zu Klagen gegen den behandelnden Arzt. Gelingt es diesem in einem möglichen Prozess nicht nachzuweisen, dass er den Patienten vor dem Eingriff hinreichend aufgeklärt hat, wird regelmäßig der Klage stattgegeben. Auch in den Fällen, in denen ein Behandlungsfehler gar nicht gegeben war, sich also z. B. nur ein typisches Risiko der Behandlung verwirklicht hat, wird wegen mangelnder Aufklärung geklagt.

Allein die Tatsache, dass der Patient wegen fehlender oder mangelnder Aufklärung nicht wirksam in den Eingriff eingewilligt hat, begründet die Haftung des Arztes. Das gilt es zu vermeiden.

Die Beweislast hinsichtlich ordnungsgemäßer Aufklärung trägt die/der Ärztin/ Arzt.

\section{Ablauf des Patientenkontaktes}

Der erste Kontakt mit dem Patienten erfolgt in Form eines Beratungsgespräches, in dem der Patient seine Wünsche und Vorstellungen bezüglich einer möglichen Haartransplantation vorträgt. Nach der klinischen Diagnosestellung erfolgt dann eine ehrliche und detaillierte Beschreibung der operativen Möglichkeiten und der zu erwartenden Ergebnisse, immer aber auch mit den Hinweisen auf eine nicht mögliche Erfolgsgarantie sowie auf die Möglichkeit der Unzufriedenheit des Patienten mit dem Endergebnis.

Arzt und Patient besprechen gemeinsam das Empfängerareal, wobei ein natürliches Ergebnis angestrebt werden sollte. Anhand einer Dichtemessung der Haarwurzelgruppen im Spenderund Empfängerareal wird der Bedarf an Transplantaten („follikular units“) ermittelt. Für das Gespräch sollte der Arzt sich genügend Zeit nehmen, um auch den genauen Ablauf des Eingriffes, mögliche Komplikationen sowie möglicherweise für den Eingriff relevante Vorerkrankungen des Patienten und damit verbundene Risiken zu besprechen.

Am Tag des Eingriffes erfolgen eine ausführliche Fotodokumentation der präoperativen Ausgangssituation sowie Anzeichnung des Empfänger- und Spenderareals in Abstimmung mit dem Patienten.

Die Haartransplantation wird von Arzt/Ärztin persönlich durchgeführt mit Unterstützung von speziell geschulten technischen Assistenten/-innen.

Nach Beendigung der Operation erfolgt eine Sicherungsaufklärung mit den empfohlenen Verhaltensregeln für die ersten postoperativen Wochen, damit die transplantierten Haarwurzeln erfolgreich anwachsen und nicht durch ein unbeabsichtigtes Verhalten, z.B. durch ein $\mathrm{zu}$ frühes Waschen der Kopfhaut, geschädigt werden.

Eine telefonische Rückfrage beim Patienten am 1. oder 2. postoperativen Tag bezüglich des Heilungsprozesses und möglicher Beschwerden dient der intensiven Patientenführung.

Ein abschließender Nachsorgetermin sollte mit dem Patienten individuell vereinbart werden. 


\section{Warum klagen Patienten?}

In den meisten Fällen beklagen die Patienten eine mangelnde Haardichte im Empfängerareal sowie entstandene sichtbare Vernarbungen.

Je weniger dringlich ein Eingriff ist, desto höher ist die Anforderung an die Aufklärungspflicht. ${ }^{1}$

Häufig ähneln Beratungsgespräche „kommerzieller Anbieter" einem Verkaufsgespräch. Dem Patienten wird suggeriert, dass er sein gewünschtes Ergebnis problemlos erhält, zum Teil für einen speziellen niedrigen „Angebotspreis“ mit „Haarwuchsgarantie“.

\section{Schlussfolgerung}

Die ordnungsgemäße Aufklärung des Patienten ist die Grundvoraussetzung dafür, dass überhaupt eine wirksame Einwilligung erteilt werden kann.
Eine juristisch wirksame Aufklärung richtet sich nach folgenden Punkten [2]:

- Wer wird aufgeklärt (Aufklärungsadressat)?

- Von wem wird aufgeklärt (Aufklärungspflichtiger)?

- Worüber wird aufgeklärt (Aufklärungsumfang)?

- Wie wird aufgeklärt (Art und Weise der Aufklärung)?

- Wann wird aufgeklärt (Aufklärungszeitpunkt)?

Bei Berücksichtigung dieser Aspekte ist eine von der Rechtsprechung geforderte ordnungsgemäße Aufklärung gewährleistet, die aus eigenem Interesse des Behandlers schriftlich in der Patientenakte detailliert festgehalten werden sollte [2].

Um vor Gericht Recht zu bekommen, ist eine schriftliche Dokumentation immer empfehlenswert und glaubhaft [2].

Hierzu sind folgende Erläuterungen wichtig:

Beim Aufklärungsadressaten muss es sich um den zu behandelnden und mündigen Patienten selbst handeln. Eine
Haartransplantation bei Minderjährigen stellt die absolute Ausnahme (z. B.: medizinisch indizierte Behandlung) dar und kann nur mit Zustimmung der Erziehungsberechtigten erfolgen.

Für eine erfolgreiche Haartransplantation ist eine korrekte Indikations- und Diagnosestellung Voraussetzung, denn nicht jeder Patient ist geeignet.

Die Aufklärung muss durch einen/eine Arzt/Ärztin, der/die sowohl über die entsprechenden Operationskenntnisse als auch über die notwendige Erfahrung zur Durchführung einer Haartransplantation verfügt, erfolgen. Grundsätzlich muss die Aufklärung mündlich in Anwesenheit von Arzt und Patient stattfinden.

Gerade in letzter Zeit im Rahmen der SARS-CoV-2-Pandemie mit Kontaktbeschränkungen wird der Ruf nach einer rechtlich ausreichenden telefonischen Beratung immer lauter. Nach einer Entscheidung des Bundesgerichtshofes von 2010 gilt, dass eine telefonische Beratung in einfach gelagerten Fällen möglich ist; dies bedarf aber der Zustimmung

Hier steht eine Anzeige. 
des Patienten. ${ }^{2}$ Unter Beachtung des Datenschutzes ist hierbei mit den aktuellen technischen Möglichkeiten eine Videokonferenz ebenfalls sinnvoll, diese ersetzt aber eine mindestens $24 \mathrm{~h}$ präoperativ durchgeführte persönliche Aufklärung nicht. Insbesondere bei rein ästhetischen Eingriffen sollte der Arzt nicht auf die schriftliche Dokumentation eines persönlich durchgeführten Aufklärungsgespräches verzichten.

Die Aufklärung muss durch eine/einen in der Haartransplantation ausgebildeten Arzt/Ärztin erfolgen. ${ }^{3}$

\section{Art und Weise der Aufklärung}

Merke: Je weniger dringlich eine Operation medizinisch gesehen einzustufen ist, desto schonungsloser muss die Aufklärung erfolgen.

Die Aufgabe der sog. Risikoaufklärung wird darin gesehen, dem Patienten schonungslos die Risiken zu nennen, die trotz fehlerfreier Durchführung der Haartransplantation möglicherweise für ihn bestehen. Auch über seltene Risiken hat der Arzt aufzuklären, wenn diese für den Eingriff spezifisch sind und die persönliche und wirtschaftliche Lebensführung des Patienten beeinflussen könnten $[2,3] .4,5$

Besonders junge Patienten mit androgenetischer Alopezie erwarten häufig eine Wiederherstellung des ursprünglichen Haarbildes. Allerdings muss bei genetischer Disposition davon ausgegangen werden, dass sich der Haarausfall bei jüngeren Patienten auch nach der Haartransplantation (HTX) noch weiterentwickeln wird.

Hierzu sollten während des Aufklärungsgespräches eine vorsichtige Prognose und die Limitation der Operation im Falle des weiteren Haarverlustes mit Ausbildung einer kompletten Oberkopfglatze erläutert werden. Eine nachhalti-

\footnotetext{
2 Aufklärung per Telefon, BGH Urteil vom 15.06.2010, Az:IVZR 204/09.

$3 \S 630$ e Abs. 2S. 1 Nr. 1 BGB.

${ }^{4}$ Gesetz zur Verbesserung der Rechte von Patientinnen und Patienten, Bundesgesetzblatt Teil 1,2013,Nr. 9 vom 25.02.2013.

5 §630cAbs.3BGB.
}

ge Planung des Operationsgebietes und des Umfangs sollte gemeinsam mit dem Patienten besprochen werden. Eine zu tief rekonstruierte Haaransatzlinie wird bei fortschreitendem Haarverlust zu einer Vergrößerung des notwendigen Behandlungsareals führen.

Empfehlenswert ist es, im Aufklärungsgespräch darauf hinzuweisen, dass keine $100 \%$ ige Dichte „wie es früher einmal war" $\mathrm{zu}$ erreichen ist und dass bei fortschreitendem Haarausfall eine weitere Haartransplantation notwendig sein kann.

Jede Operation, sei sie noch so minimal-invasiv, hinterlässt Narben. Sicherlich wird jeder Operateur so handeln, dass diese möglichst unauffällig verheilen. Jedoch können auch nach Haartransplantationen sowohl in der FUT - als auch in der FUE-Technik zum Teil deutlich sichtbare Narben entstehen. Dabei werden regelmäßig psychische Auswirkungen der sichtbaren Narbe mit sekundärem Schamgefühl und entsprechenden Problemen im privaten und beruflichen Alltag beklagt. Deshalb sollten große sichtbare Narben durch eine sorgfältige Planung und durch eine optimierte Entnahme- bzw. Nahttechnik minimiert werden. Beispielsweise können bei der Streifenentnahme unter Verwendung der sog. ,Trichophytic-closure-Technik“, bei der die Hautschichten tangential inzidiert werden, damit Haare durch die Naht wachsen und die eigentliche Narbe kaschiert wird, auffällige Narbenbilder vermieden werden. Bei der FUE-Methode gilt dies für eine gleichmäßige und nicht zu dichte Entnahme mit möglichst kleinen Bohrhülsen.

Auf trotz aller Sorgfalt entstehende sichtbare Narben sollte im Aufklärungsgespräch explizit dennoch hingewiesen werden.

\section{Aufklärungsumfang}

Ebenso besteht eine wirtschaftliche Aufklärungspflicht des Arztes. Der Patient ist über die Behandlungskosten (Honorarvereinbarung) und über mögliche Folgekosten aufzuklären, wenn keine Kostenübernahme durch die Krankenkassen erfolgt.
Typischerweise kann dies der Fall sein bei androgenetischer Alopezie, die die häufigste Indikation zur Haartransplantation ist. Diese genetische Disposition ist ein fortschreitender Prozess, der bereits ab dem 20. Lebensjahr auftreten kann und sich bis zum ca. 50. Lebensjahr in unterschiedlichem Ausmaß weiterentwickeln kann. Das muss besonders jüngeren Patienten erklärt werden mit entsprechenden Konsequenzen. Ein junger Patient, der sich einer Haartransplantation unterziehen möchte, muss bei androgenetischer Alopezie dringlich darauf hingewiesen werden, dass ohne eine medikamentöse Unterdrückung des weiteren Haarverlustes ggf. eine zweite oder sogar dritte Operation notwendig sein kann, um dauerhaft das gewünschte Ergebnis $\mathrm{zu}$ erreichen. Dadurch können weitere Kosten für den Patienten entstehen.

Unrealistische Erwartungen des Patienten oder nicht ausreichendes Spenderhaar sollten ggf. Gründe sein, die Haartransplantation abzulehnen.

Hierbei ist es im Rahmen des Erstgespräches ebenfalls wichtig, den Patienten nicht nur über die verschiedenen Operationstechniken der Haartransplantation (FUT und FUE), sondern auch über alternative Behandlungsmöglichkeiten $\mathrm{zu}$ unterrichten (z. B.: Toupet/künstliche Haarteile, Rasur/Shaved-Look, Scalpmikropigmentierung oder medikamentöse Behandlung).

Die Aufklärung über unterschiedliche Behandlungsalternativen dient dem Selbstbestimmungsrecht des Patienten und ist daher Voraussetzung einer rechtmäßigen Behandlung. ${ }^{6}$

Auch die Darlegung des Ablaufes der Operation über Dauer, Art der Betäubung, evtl. Medikationen während des Eingriffs, geplante Pausen bei längeren Prozeduren sowie das weitere Vorgehen im Anschluss an die Behandlung sollte im Rahmen einer Sicherungsaufklärung/ therapeutischen Aufklärung erwähnt und schriftlich dokumentiert werden. Hierbei ist auch über eine nach dem Eingriff bestehende Fahruntüchtigkeit oder über Ausfallzeiten für den Patienten zu informieren.

\footnotetext{
${ }^{6}$ BGH, Urteil vom 15.03.2005, AZ:VIZR 313/03.
} 
Um in der alltäglichen Praxis keine wichtigen Komplikationen zu vergessen, können computergestützte Präsentationen oder Aufklärungsvordrucke (z.B.: Pro Compliance, Diomed, Perimed) hilfreich sein. Insbesondere bei rein ästhetischen Operationen, die allein eine Verbesserung des äußeren Erscheinungsbildes zum Ziel haben, ist es ratsam, nicht nur Lichtbilder von erfolgreichen Behandlungen zu zeigen, sondern auch Bilder von Operationen, die nicht zum gewünschten Erfolg oder sogar zu Komplikationen geführt haben.

Fotos von unterschiedlich gelungenen oder auch misslungenen Operationsergebnissen zu zeigen hilft, die Erwartungen des Patienten auf ein realistisches Niveau zu bringen.

\section{Aufklärungszeitpunkt}

Die Aufklärung sollte mit einem zeitlichen Abstand zum Eingriff selbst stattfinden. Das Selbstbestimmungsrecht des Patienten fordert die Rechtzeitigkeit der ärztlichen Aufklärung, damit dieser Vorund Nachteile ohne Zeitdruck abwägen kann [2].

Bei ambulanten Eingriffen muss die Aufklärung nach Rechtsprechung mindestens $24 \mathrm{~h}$ vorher erfolgen. Gemäß den Leitlinien des Verbandes Deutscher Haarchirurgen (VDHC) sollte sichergestellt werden, dass der Patient sich mindestens 4 bis 6 Wochen vor dem Eingriff mit der Thematik befasst hat bzw. schon vorbehandelt ist oder schon vorher Beratungen eingeholt hat. Eine persönliche Aufklärung sollte spätestens $24 \mathrm{~h}$ vor der Haartransplantation stattgefunden haben [4].

Um als Behandler im Falle einer gerichtlichen Auseinandersetzung eine ausführliche Aufklärung beweisen $\mathrm{zu}$ können, ist eine individuelle schriftliche Dokumentation über Zeitpunkt des Gespräches, Gesprächsinhalt, Anamneseerhebung, Untersuchungsbefunde mit Diagnosestellung und Operationsplanung, individuelle Gegebenheiten und Wünsche des Patienten und Einwilligung des Patienten ratsam.

J Ästhet Chir 2021 · 14:108-112 https://doi.org/10.1007/s12631-021-00266-w

(c) Springer Medizin Verlag GmbH, ein Teil von Springer Nature 2021

\section{A. Hortling}

\section{Gutachten nach Haartransplantation. Auf was sollte man in der präoperativen Aufklärung achten?}

\section{Zusammenfassung}

Die Eigenhaarverpflanzung erfreut sich zunehmend einer gesteigerten Nachfrage. Es handelt sich um einen risikoarmen Eingriff mit einem großen Benefit für die Patienten. Um mögliche Klagen und um postoperative Unzufriedenheiten der Patienten und Patientinnen zu vermeiden, ist neben der korrekten Durchführung der Prozedur eine umfassende und ausführliche Aufklärung im Vorfeld notwendig. Insbesondere, da derzeit ein definierter Facharztstandard zur Durchführung einer Haartransplantation fehlt, ist die Einhaltung einer formal vollständigen
Vorbereitung einschließlich einer möglichst umfassenden Beratung im Vorfeld der geplanten Haartransplantation zu empfehlen. Der Beitrag soll Tipps und Anleitung geben, um typische Unzufriedenheiten und auch gerichtlich vorgebrachte Vorwürfe zu vermeiden.

Schlüsselwörter

Sicherungsaufklärung · Patientenwünsche · Follicular-unit-excision-Technik · Follicularunit-Transplantation · Eigenhaarverpflanzung

\section{Expert assessment after hair transplantation. What should be included in the preoperative education?}

\section{Abstract}

The demand for autologous hair transplantation is steadily increasing. The procedure has a low risk for complications with a great benefit for the patients. In order to prevent complaints and legal actions as well as postoperative dissatisfaction of the patients, in addition to the correct performance of the procedure a comprehensive and extensive clarification is necessary in advance. In particular, as a defined specialist standard for the performance of hair transplantation procedures is lacking, the compliance with a formal complete preparation including

\begin{abstract}
a thorough and complete informed consent as well as a preoperative consultation are recommended in advance of a planned hair transplantation. In this article tips and guidance are given on how to avoid typical grounds for dissatisfaction and also legal claims on behalf of the patients.
\end{abstract}

Keywords

Back-up informed consent - Patient wishes . Follicular unit extraction technique - Follicular unit transplantation - Autologous hair transplantation

\section{Fazit für die Praxis}

- Eine ehrliche und ausführliche Aufklärung ist nicht nur die rechtliche Grundlage, sondern auch die Basis eines guten Vertrauensverhältnisses zwischen Arzt und Patienten.

- Aus einer korrekten Aufklärung muss hervorgehen „wer, von wem, über was, wann" aufgeklärt wurde.

- Empathie und Vertrauen sind die Grundlage eines guten PatientenArzt-Verhältnisses.

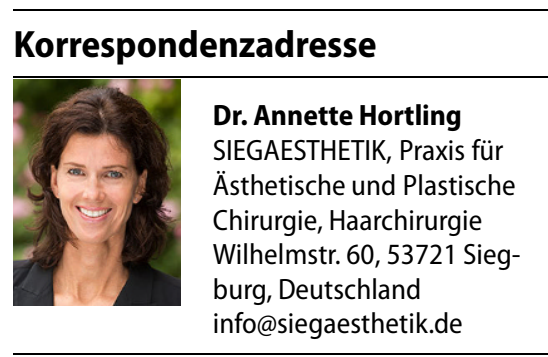

Einhaltung ethischer Richtlinien

Interessenkonflikt. A. Hortling gibt an, dass kein Interessenkonflikt besteht.

Dieser Beitrag beinhaltet keine Studien an Menschen oder Tieren. Alle Patienten, die über Bildmaterial oder anderweitige Angaben innerhalb des Manuskripts 
zu identifizieren sind, haben hierzu ihre schriftliche Einwilligung gegeben.

\section{Literatur}

1. https://ishrs.org/wp-content/uploads/2020/05/ Report-2020-ISHRS-Practice-Census-05-22-20. pdf.Zugegriffen: 24. März 2021

2. von Heimburg, Richter, Lemperle - Ästhetische Chirurgie 36. Erg. Lfg. 9/16, Grundlagen der Aufklärung und Einwilligung bei chirurgischen Eingriffen

3. Schröder B, Teichner M (2009) Die wirtschaftliche Aufklärungspflicht bei medizinisch nicht indizierten und ästhetischen eingriffen. Plast Chir $4: 224$

4. https://www.vdhc.de/downloads/VDHCLeitlinie_HT.pdf.Zugegriffen:24.März2021

\section{Brustkrebs: Depression als Begleiterkrankung}

Auch noch viele Jahre nach der Therapie

Selbst nach einer erfolgreichen Therapie sind viele Brustkrebspatientinnen noch über lange Zeit sehr belastet. Wissenschaftler im Deutschen Krebsforschungszentrum (DKFZ) haben in einer Studie mit insgesamt mehr als 4.000 Frauen belegt, dass gerade Patientinnen, deren Therapie bereits 5 bis 15 Jahre zurückliegt, häufig mit Depressionen kämpfen. Das zeigt, wie wichtig es ist, die psychische Verfassung bei der Behandlung betroffener Frauen nicht zu vernachlässigen.

Brustkrebs ist nach wie vor die häufigste Krebserkrankung bei Frauen. Weltweit diagnostizieren Ärzte jedes Jahr mehr als zwei Millionen Neuerkrankungen; allein in Deutschland erhalten jährlich rund 69.000 Patientinnen diese Diagnose. Gleichzeitig verbessern sich aber auch die Überlebenschancen dank immer besserer Diagnostik und Therapie. Doch auch nach einer erfolgreichen Behandlung ist die Lebensqualität vieler Betroffener noch lange Zeit eingeschränkt. Sie leiden etwa unter dem chronischen Erschöpfungssyndrom Fatigue oder unter therapiebedingten Muskel- und Gelenkschmerzen. Auch über Depressionen klagen viele Betroffene.

„Während das Auftreten von Depressionen innerhalb der ersten fünf Jahre nach der Brustkrebstherapie bereits recht gut untersucht ist, ist über die Häufigkeit von Depressionen bei Langzeitüberlebenden bislang wenig bekannt", sagt Volker Arndt, Epidemiologe im Deutschen Krebsforschungszentrum. „Wir wollten wissen, welche Rolle Depressionen bei Patientinnen auch noch viele Jahre nach der erfolgreichen Krebsbehandlung spielen, und welche Faktoren dies möglicherweise beeinflussen."

Die DKFZ-Epidemiologen um Arndt untersuchten insgesamt 3.100 Brustkrebsüberlebende, deren Therapie zwischen 5 und 16 Jahre zurücklag, auf Anzeichen einer Depression. Zum Vergleich schlossen sie 1.005 Frauen ohne eine entsprechende Krebserkrankung in ihre Studie ein. „Wir haben festgestellt, dass Langzeitüberlebende, deren Therapie bereits zwischen 5 und 15 Jahren zurückliegt, häufiger unter Depressionen leiden als Frauen, die nie an Brustkrebs erkrankt waren", sagt Erstautorin Daniela Doege. Insbesondere betroffen waren Frauen, bei denen die Krebserkrankung wiedergekehrt war oder bei denen Metastasen festgestellt wurden. Weitere Risikofaktoren waren höheres
Alter, Übergewicht, sowie eine eingeschränkte oder aufgegebene Berufstätigkeit. „Wie die einzelnen Faktoren das Depressionsrisiko beeinflussen, können wir auf der Grundlage unserer Studie allerdings nicht erklären", sagt Doege.

Doch auch wenn sich die exakten Ursachen für das erhöhte Depressionsrisiko derzeit nicht ausmachen lassen, enthält das Studienergebnis eine bedeutende Botschaft: "Unsere Daten zeigen wie wichtig es ist, dass behandelnde Ärzte bei Brustkrebspatientinnen, gerade bei Betroffenen mit Metastasen oder wiederkehrenden Tumoren, nicht nur die rein onkologischen Symptome therapieren“, sagt Arndt. „Entscheidend ist auch, die psychische Verfassung der Betroffenen im Blick zu behalten und bei Bedarf Hilfe anzubieten."

Literatur: Daniela Doege, Melissa S Y Thong, Lena Koch-Gallenkamp, Lina Jansen, Heike Bertram, Andrea Eberle, Bernd Holleczek, Ron Pritzkuleit, Annika Waldmann, Sylke R Zeissig, Hermann Brenner, Volker Arndt: Age-specific prevalence and determinants of depression in long-term breast cancer survivors compared to female population controls Cancer Medicine 2020, DOI: 10.1002/cam4.3476

Quelle: Deutsches Krebsforschungszentrum 